\title{
Eine große Erzählung von Mexiko-Stadt
}

Rezension zu Streule, Monika (2018): Ethnografie urbaner Territorien. Metropolitane Urbanisierungsprozesse von Mexiko-Stadt. Reihe Raumproduktionen: Theorie und gesellschaftliche Praxis Band 32. Münster: Westfälisches Dampfboot.

Abb. 1 Titel des Buches (Quelle: Westfälisches Dampfboot)

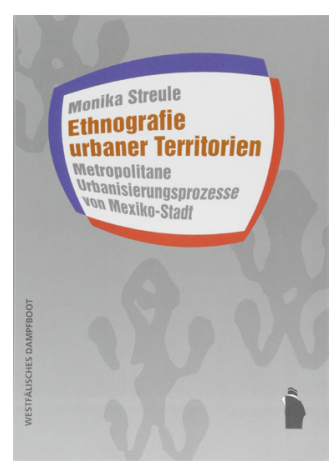

"It is not enough simply to study the cities of the global South as interesting, anomalous, different, and esoteric empirical cases"(Roy 2009: 820) - eine nach wie vor aktuelle Forderung. Ananya Roy und anderen Autor_innen folgend muss es daher darum gehen, Theorien und Konzepte vom Süden her zu denken, um die Rollenaufteilung des globalen Nordens als Theorieproduzentin und des globalen Südens als empirischem Feld zu überwinden (Huffschmid/Wildner 2013: 17). Dem entspricht die Forderung einer dezentralisierten Theorie- und Begriffsbildung, um etwa im lateinamerikanischen Kontext entwickelte Konzepte in die internationale Stadtforschung mit einzubringen und deren Relevanz für Untersuchungen andernorts zu prüfen (vgl. Lanz 2015: 124).

Den lokalen Kontext und die Spezifik lateinamerikanischer Städte etwa machen Anne Huffschmid und Kathrin Wildner in vier Dimensionen aus (2013: 12ff.): im Palimpsest-Charakter mit architektonischen Layern unterschiedlicher Zeiten; in der kulturellen Heterogenität und Hybridisierung; in einer ausgeprägten Kultur des öffentlichen Raums; und, angelehnt an Ananya Roy, in der Informalität als Regulationsform. Im lateinamerikanischen Zusammenhang entwickelte Konzepte wie das des Territoriums als Kategorie von Bewegung (ebd.: 19) oder vom imaginario als „Verschränkung von subjektiver Raumwahrnehmung und -erfahrung, Diskursen und Narrationen mit der sozialen Organisation des städtischen Alltaglebens“" (ebd.: 21) sehen die Autorinnen als mögliche Inspirationsquellen für die internationale Stadtforschung. An ihnen setzt auch Monika Streule an.

Die hier vorgestellte Arbeit bewegt sich in einem Feld der Stadtforschung, das idealerweise disziplinübergreifend zu bearbeiten sei (Huffschmid/ Wildner 2013: 13ff.), in dem aber ethnographischen Ansätzen besondere Bedeutung zukommt (vgl. ebd.: 14 sowie Streule 2018: 18). Streule hat sich ebenfalls für eine ethnographische Herangehensweise an Urbanisierungsprozesse in Mexiko-Stadt entschieden. Im Mittelpunkt ihrer Untersuchung stehen urbane Transformationsprozesse (13), ihr Ziel ist ,eine differenzierte Repräsentation von Mexiko-Stadt als hybrides, dynamisches, umkämpftes und widersprüchliches urbanes Territorium“ (14). Dafür fragt Streule nach 
den beteiligten Subjekten, den materiellen Spuren, lokalen Folgen sowie den Auswirkungen auf das Alltagsleben der Bewohner_innen (15). Die Autorin stellt dieser umfassenden Forschungsfrage die Prämisse von Territorium als gesellschaftlichem Produkt voran, das wiederum als Ausgangsbasis für die Analyse gesellschaftlicher Verhältnisse dienen kann (77). Das so beschriebene Feld empirisch zu durchdringen stellt sich als große Herausforderung heraus, wie ich nach einer kurzen Skizze der Arbeit erläutern möchte. Den Schwerpunkt dieser Rezension lege ich aus diesem Grund (sowie aufgrund persönlicher Interessen) auf methodologische Fragen, die Streules Arbeit meiner Meinung nach aufwirft. Dessen unbenommen argumentiere ich zum Ende des Beitrags, dass Streule mit ihrer Dissertation ein großer Wurf gelingt.

Der empirische Teil der Arbeit ruht auf zwei Pfeilern: erstens auf einer Thesenkarte, die als zentrales Ergebnis der Untersuchung die textliche Darstellung der empirischen Erkenntnisse strukturiert. Diese bestehen aus recorridos explorativos (Wahrnehmungsspaziergängen) und entrevistas en movimiento (Interviews in Bewegung). Zweitens fußt die Arbeit auf einer historischen Analyse, mit der Streule Henri Lefebvres Idee einer regressiv-progressiven Analyse umsetzt (77ff.). Die Karte, die die Autorin als „territorialbezogene grafische Abstraktion sozialer und räumlicher komplexer Wirklichkeiten der untersuchten Urbanisierungsprozesse“ (46f.) versteht, liegt dem Buch bei.

Nachdem Streule bereits in der Hinführung auf die Notwendigkeit einer historischen Kontextualisierung hinweist, wie sie auch der argentinisch-mexikanische Stadtforscher García Canclini fordert (70, vgl. auch Wildner/Huffschmid 2013: 12), entfaltet sie zunächst eine umfassende Periodisierung der Urbanisierungsgeschichte Mexiko-Stadts. Während sie im methodologischen Abschnitt zu diesem Analyseschritt ihren Quellenkorpus nicht näher spezifiziert, wird bei der Lektüre des Kapitels deutlich, dass es sich um eine Analyse von Sekundärliteratur handelt, die sie nach den Prinzipien der regressiv-progressiven Analyse nach Lefebvre aufbaut. Ihr Ziel ist es, historische Narrative zu rekonstruieren und auf die Simultanität verschiedener Prozesse hinzuweisen. Von der aztekischen Hauptstadt Tenochtitlan führt ihre Erzählung über die Kolonialisierung bis zum jüngsten Urbanisierungsregime der zunehmenden Polarisierungstendenzen und einer lauter werdenden außerparlamentarischen Opposition. Für alle Regime erläutert sie anschaulich die historischen Voraussetzungen und macht die Leser_innen mit wichtigen Akteur_innen und Prozessmerkmalen bekannt. In diesem Teil gelingt es Streule, eine strukturierte und schlüssige Historisierung zu entwickeln, dabei aber auch auf Widersprüche und Gleichzeitigkeiten hinzuweisen. Die Stadt liest sie als Palimpsest: in der heute vorhandenen materiellen Struktur seien hegemoniale Prozesse aus verschiedenen Zeiten eingeschrieben (88).

Nach dieser historischen Kontextualisierung folgen Erläuterungen zu neun Urbanisierungsprozessen, die Streule heute in Mexiko-Stadt ausmacht - und die sie in der Thesenkarte visualisiert und lokalisiert. Ziel ist zu zeigen, wie Territorien produziert werden, die sich wiederum auf Alltagsebene auswirken (190). $\mathrm{Zu}$ den genannten Prozessen gehören die Industrialisierung und Urbanisierung von Vororten wie Ixtapalupa und Xochimilco, oder die Konsolidierung einst informeller Siedlungsgebiete, etwa in Iztapalapa (40). Anschließend skizziert Streule allgemeine Tendenzen in Mexiko-Stadt, die 
das heutige Urbanisierungsregime insgesamt ausmachen. Hier identifiziert sie etwa die ,Eventisierung' der Stadtpolitik und eine marktförmige Urbanisierung, die Privatisierung und Militarisierung des öffentlichen Raums sowie Ermächtigungsstrategien von Seiten der Bewohner_innen (304ff.).

Während diese empirischen Ausführungen überaus instruktiv sind, stellen sich bei den methodologischen Erklärungen verschiedene Fragen. So holt Streule gleich zu Beginn zum Rundumschlag aus und kritisiert, dass es bislang nicht gelungen sei, der Komplexität aktueller Urbanisierungsprozesse empirisch gerecht zu werden (14). Mit der allgemein gehaltenen (und leider nicht weiter belegten) Kritik legt Streule die Messlatte hoch, der sie mit einer qualitativen Untersuchung begegnen möchte, um die Alltagsebene der Bewohner_innen erfassen zu können (14). Die Beziehung zwischen strukturellen Prozessen, der Material- und Subjektebene konzipiert sie knapp mit Lefebvres triadischem Raum (12). Wichtig ist ihr außerdem der Maßstabssprung von den administrativen Grenzen Mexiko-Stadts auf die Metropolebene, die den umliegenden Bundesstadt Estado de México miteinschließt. Denn ethnographische Studien würden sich bislang lediglich mit kleinteiligen Räumen (19) befassen. Auch hier fehlen die Belege und überzeugende Erklärungen, welchen Vorteil die Entnahme von „Proben“ (19) auf Metropolebene hat (auf der sie letztlich ein neues „,vermeintlich überblickbares Untersuchungsgebiet“ (ebd.) schafft). Auch mit ,instrumentell konnotierten“ Ethnographien, denen sie Körperlosigkeit und Verflachung unterstellt, geht sie hart ins Gericht (18). Solche Kritiken wären überzeugender, wenn sie mit Beispielen oder detaillierten Erklärungen versehen worden wären.

Methodologisch fehlt eine Konzeption der Beziehung zwischen den in Interviews getätigten Aussagen, ihren Notizen und der Alltagsebene der Befragten. Sie verweist an mehreren Stellen auf „die“ Grounded Theory (18, 24 und 31), nicht aber auf eine entsprechende Auswertung, die in einer auf Grounded Theory basierten Untersuchung etwa eine detaillierte Codierung, die Erstellung von Memos und die Herausarbeitung von Kategorien umfassen könnte. Streule spricht von Feld- und Gesprächsnotizen, deren Erstellung ein subjektiv geprägter Prozess sei und bemerkt, dass sich Datenerhebung und -interpretation nicht unterscheiden ließen (43). Damit folgt sie zwar dem qualitativen Konzept eines iterativ-zyklischen Untersuchungsablaufs. Auf welche Weise aber eine empirische Sättigung im Sinne einer intensiven Datenanalyse (Strübing et al. 2018: 90) und damit eine Entfremdung vom Material stattfand, lässt sich aus diesen Ausführungen nicht erschließen. Der Verweis auf die Illusion „authentischer und wertfreier Rohdaten“ (43) lenkt davon ab, dass die Frage wenigstens zu reflektieren ist, inwiefern und an welcher Stelle der Analyse der eigene Blick das Material bereits vorstrukturiert. Dazu bedarf es detaillierter methodologischer Ausführungen, die eine Selbstreflexion sicherstellen (43) und zentrale Entscheidungsmomente nachvollziehbar machen. An vielen Stellen bleiben diese Erörterungen jedoch aus, wie etwa bei der Beschreibung des Zustandekommens der Thesenkarte (44ff.), die sie schrittweise und triangulativ auf Grundlage verschiedener empirischer Grundlagen entwickelt. Unter anderem wären konkrete Beispiele, etwa für Probleme während der Auswertung, hilfreich gewesen, um den Weg von der Feldnotiz zur Farbschraffierung in der Karte verständlich zu machen. Daneben bewegt sich Streule nicht immer leicht nachvollziehbar zwischen 
den Analyseebenen, sodass etwa im Fall der Thesenkarte die Frage aufkommt, wie diese gleichzeitig untrennbar mit Streules Person verbunden und eine „Repräsentation der wichtigsten räumlichen Prozesse des ausgewählten Forschungsfelds zu einem bestimmten Zeitpunkt“ (47) sein kann.

Streules Ausführungen zur Selbstverortung (20ff.) hätten meinem Empfinden nach ebenfalls ausführlicher ausfallen können. Da sie zuvor schon auf postkoloniale Theorien Bezug genommen hatte (etwa in der Fußnote auf Seite 65), hatte ich in diesem Abschnitt eine Verortung im Feld post- und dekolonialer Ansätze erwartet, wie sie etwa 2015 in sub \urban diskutiert wurden (Debatte Band 3, Heft 1). Stattdessen verbleibt Streule bei einigen sehr allgemeinen Hinweisen zur Genese und dem Umfeld ihrer Forschung in Mexiko-Stadt sowie sehr knappen Ausführungen zu ihrer Position als „weiße mitteleuropäische Akademikerin“ (22). Diese würde ihr eine gewisse Vogelfreiheit und damit Zugang zu unterschiedlichen Lebenswelten geben (ebd.). Was das genau für ein Zugang sein mag, genauso wie die Erläuterung, welche Bedrohungskategorien es gebe und warum sie selbst aus den meisten herausfalle (ebd.), bleibt bei aller berechtigten Kritik an ausufernden Selbstreflektionen (vgl. 20) offen. Nicht zuletzt stelle ich mir die Frage, inwiefern sich der nur selten durchbrochene auktoriale Narrationsstil Streules mit einer an der Alltagswelt orientierten Forschung verträgt. Denn ohne die Konzepte mit einem geeigneten Verfahren tatsächlich aus dem Material heraus zu entwickeln oder die Befragten konsequent an der Entwicklung der Thesen zu beteiligen, bleiben die Gesprächspartner_innen wohl doch eher Informant_innen und Kommentator_innen für die von Streule vorgenommene Analyse von Urbanisierungsprozessen, als dass hier Alltagswelten rekonstruiert wurden.

Mit der beiliegenden Karte, dem zentralen Produkt der Arbeit, schafft Streule einerseits ein eingängiges visuelles Wissenschafts-Imaginario von Territorien innerhalb der Stadtregion (vgl. 69). Dem textlichen Teil der Arbeit zieht sie damit andererseits den Stachel, da die farbig eingetragenen ,dominanten' Prozesse (rosarote Bereiche metropolitaner Zentralitäten, grüne Zonen urbanisierter Dörfer, gelbe Industrieregionen etc.) zumindest visuell die Antagonismen verdecken, die sie selbst regelmäßig betont (siehe oben). Das ist jedenfalls dann der Fall, soll die Karte wie von der Autorin als Repräsentation von Urbanisierungsprozessen und nicht von Narrativen verstanden werden. Streule selbst bezeichnet ihre Methoden als experimentell (24ff.), und sie haben meines Erachtens auch das Potential, den qualitativen Methodenkanon zu erweitern sowie bestehende Konzepte wie den go-along zu verfeinern. Mit den offenen Fragen, die die Arbeit stellenweise hinterlässt und auf die Streule selbst verweist (66), trägt sie zur Methodendiskussion wertvolles ,Futter bei.

Abschließend möchte ich festhalten, dass die methodologischen Desiderata nicht im Widerspruch zu dem insgesamt überzeugenden Narrativ stehen, das Streule über Mexiko-Stadt entwickelt. Liest man diese Arbeit als regulationstheoretisch informierte und empirisch unterfütterte Erzählung über Mexiko-Stadt mit kritischem Impetus, dann schafft sie es, „zu einer Welt urbaner Phänomene [zu] sprechen“ (311). Denn die Arbeit ist sowohl anschlussfähig an eine translokale Theoriebildung als auch an eine dezentralisierte Begriffsbildung (Lanz 2015: 124). Das imaginario (Huffschmid/ 
Wildner 2013: 20f.), das Streule eng an den Begriff des Territoriums knüpft (75), ist eines der lateinamerikanischen Konzepte, das hier etwa Eingang findet. Streule schafft damit ein wichtiges empirisches Referenzwerk für den deutschsprachigen Raum, an dem sich künftige Mexiko-Stadt-Forscher_innen werden abarbeiten können. Sie skizziert die identifizierten Prozesse dicht, detail- und kenntnisreich. An dieser Stelle ist auch das mehr als umfassende Datenmaterial zu betonen, das in die Arbeit eingeflossen ist. Das besteht nicht nur aus den Feld- und Gesprächsnotizen, sondern aus einem großen Pool an wissenschaftlicher Literatur, Filminterpretationen und Zeitungsartikeln (86). Sie beschreibt in den empirischen Kapiteln sehr anschaulich, welchen Einfluss etwa verschiedene Finanzakteure oder einzelne Politiker_innen auf die Stadtpolitik nahmen und nehmen, und wie ökonomisch gut und weniger gut situierte Stadtbewohner_innen mit der marktförmigen Urbanisierung umgehen. Mit diesen inhaltlichen und außerordentlich reichen Erörterungen bietet Streule einen fundierten Ansatzpunkt für vergleichende Untersuchungen von Urbanisierungsprozessen.

\section{Autor_innen}

Giulia Montanari ist Sozialgeografin mit Schwerpunkt auf qualitativen und insbesondere visuellen Methodologien in der Geographie sowie Stadt- und Familienforschung. gmontanari@gmontanari.de

\section{Literatur}

Huffschmid, Anne / Wildner, Kathrin (2013): Das Urbane als Forschungsfeld: Öffentlichkeit, Territorien, Imaginarios. In: Anne Huffschmid / Kathrin Wildner (Hg.): Stadtforschung aus Lateinamerika - Neue urbane Szenarien: Öffentlichkeit - Territorialität - Imaginarios. Bielefeld: transcript, 9-28.

Lanz, Stephan (2015): Pluralistisches Theoretisieren der Stadt und ihrer Verknüpfungen mit dem Kolonialen - Replik. In: sub \urban 3/1, 119-128.

Roy, Ananya (2009): The 21st-century metropolis: New geographies of theory. In: Regional Studies 43/6, 819-830.

Streule, Monika (2018): Ethnografie urbaner Territorien. Metropolitane Urbanisierungsprozesse von Mexiko-Stadt. Reihe Raumproduktionen: Theorie und gesellschaftliche Praxis Band 32. Münster: Westfälisches Dampfboot.

Strübing, Jörg / Hirschauer, Stefan / Ayaß, Ruth / Krähnke, Uwe / Scheffer, Thomas (2018): Gütekriterien qualitativer Sozialforschung - Ein Diskussionsanstoß. In: Zeitschrift für Soziologie 47/2, 83-100. 